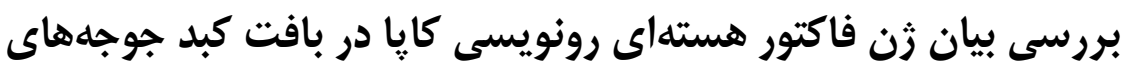

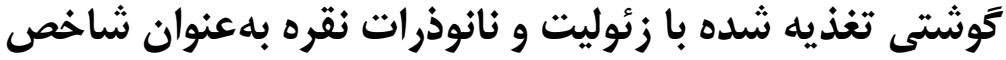

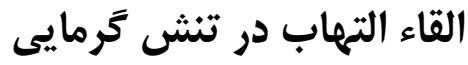

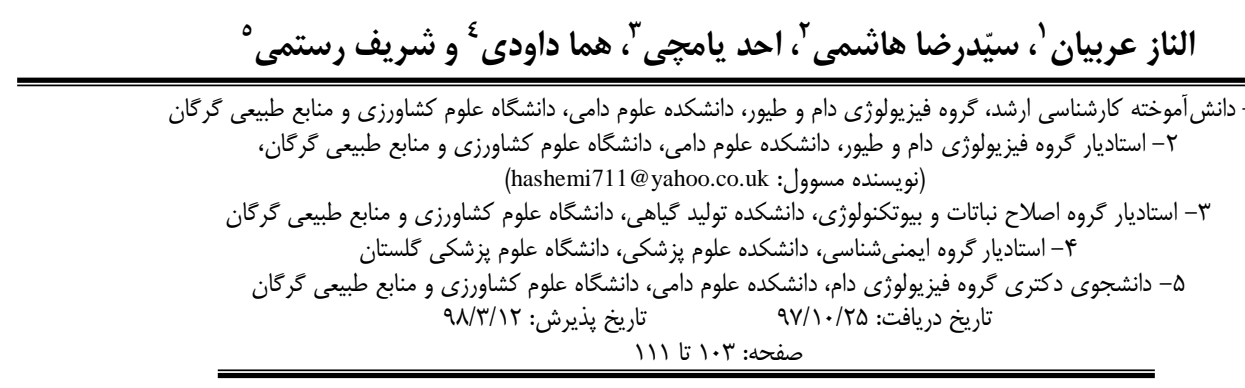

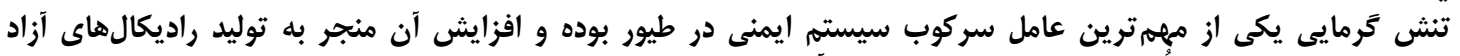

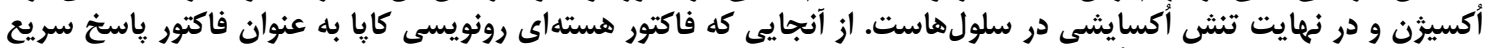

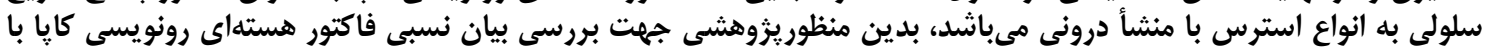

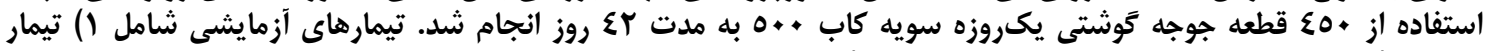

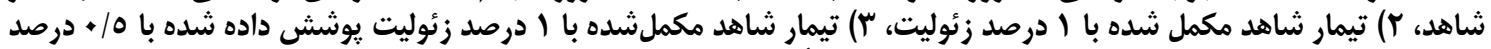

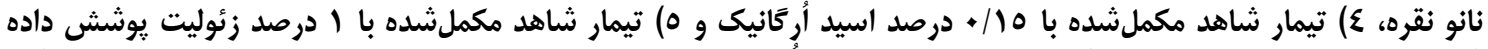

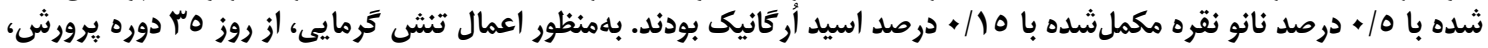

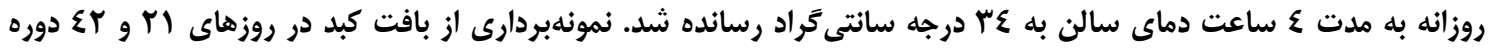

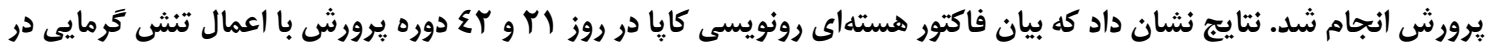

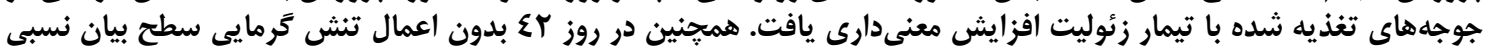

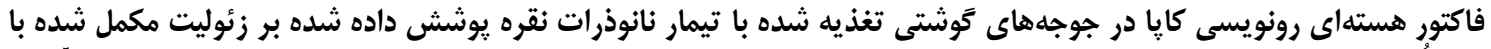

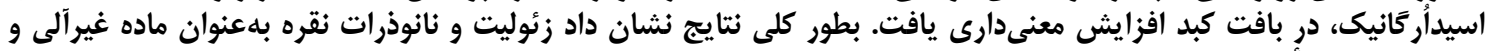

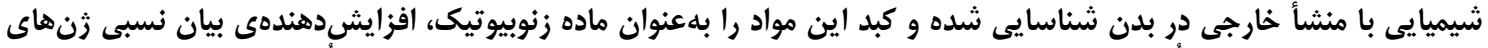

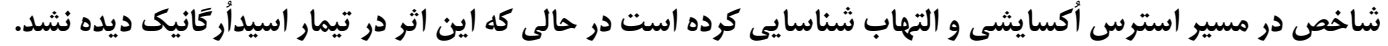

وازههاى كليدى: نانو نقره، التهاب، فاكتور هستهاى رونويسى كايا، ايمنى، جوجه توشتى

بيمارى آرزريا كه از رسوب دادن نقره ناشى مي شيود و تماس

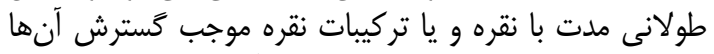

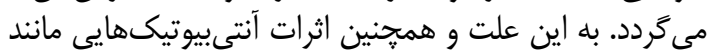

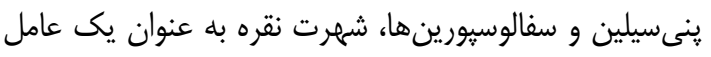

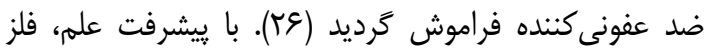

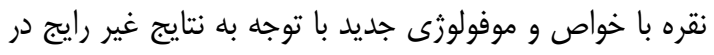

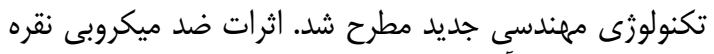

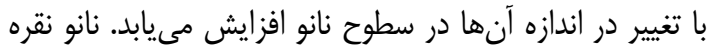

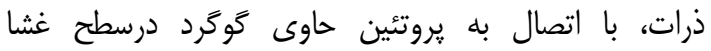

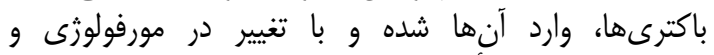

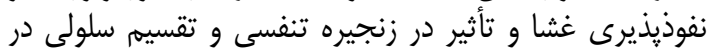

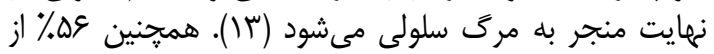

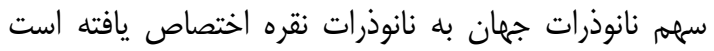

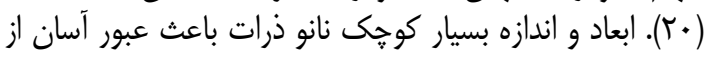

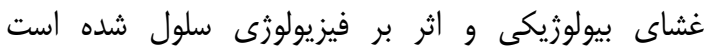

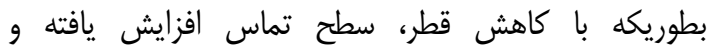

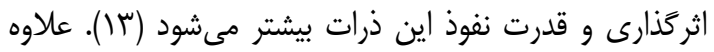

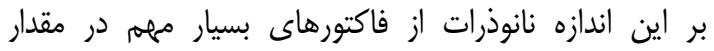

مقدمه

فن آورى نانو، دانش شناسايى و سنتز مواد در ابعادى بين

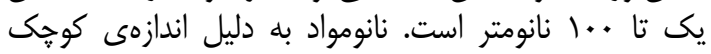

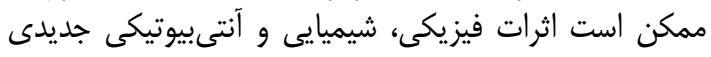

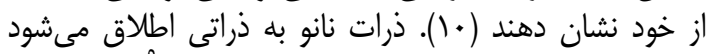

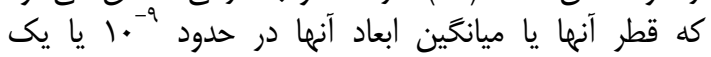

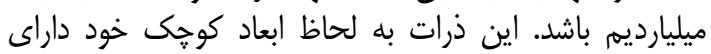

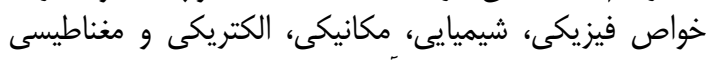

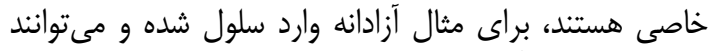

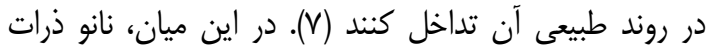

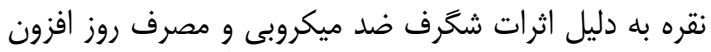

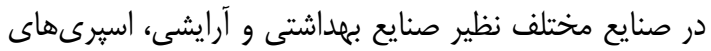

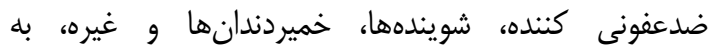

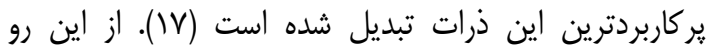

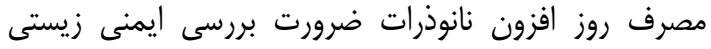

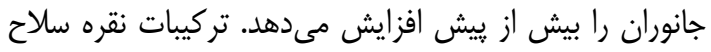

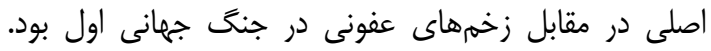

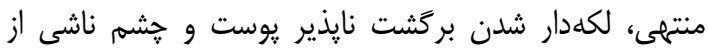




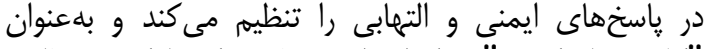

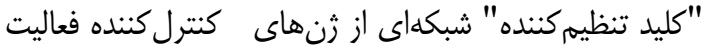

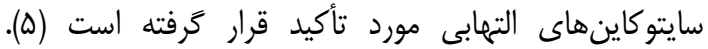

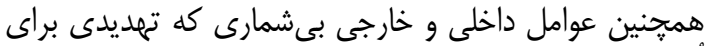

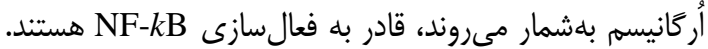

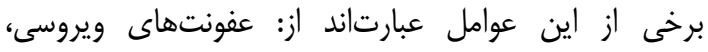

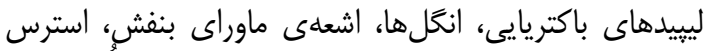

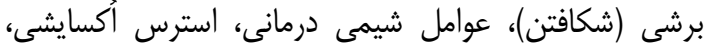

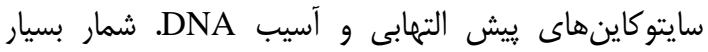

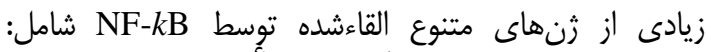
(زنهاى ويروسى (CMV) (كيرندهى

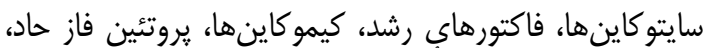

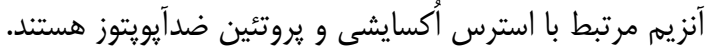

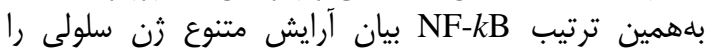

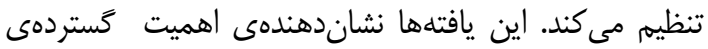

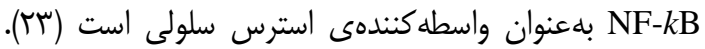

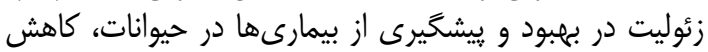

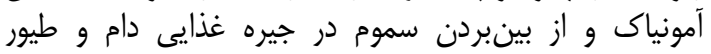

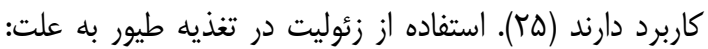

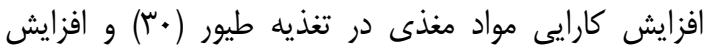

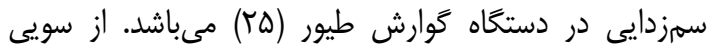

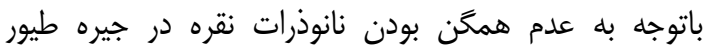

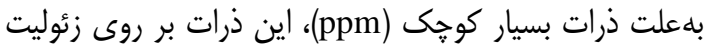

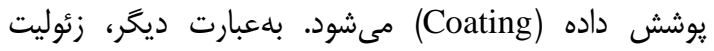

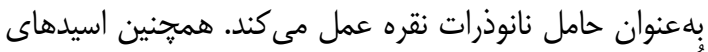

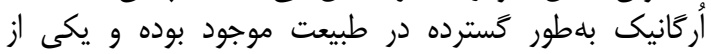

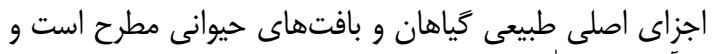

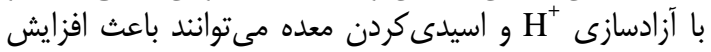

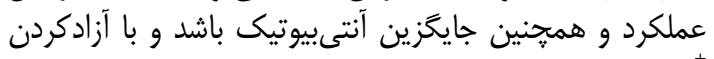

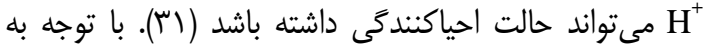

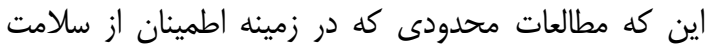

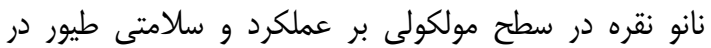

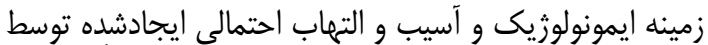

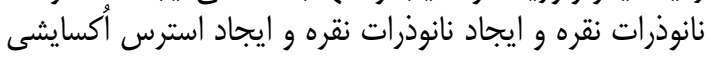

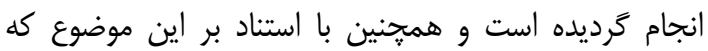

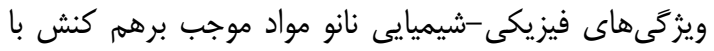

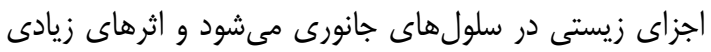

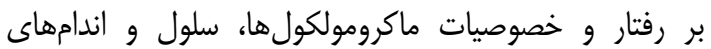

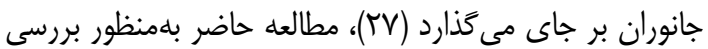

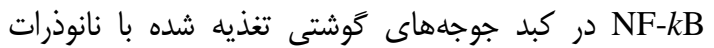
نقره يوشش داده شده بر زئوليت انجام شد.

\section{مواد و روشها}

تحقيق حاضر در ايستخاه تحقيقات طيور دانشكده علوم دامى دانى

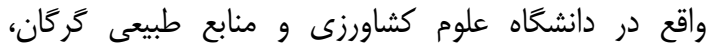

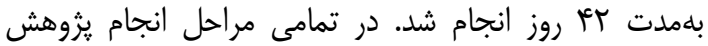

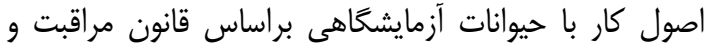
استفاده از حيوانات آزمايشخاهى ازئ كميته اخلاق كار بران بروهش
سميت ايجاد شده و توانايى هدف قرار دادن سلولها بهشمار

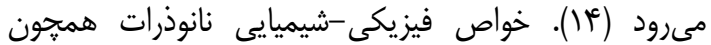

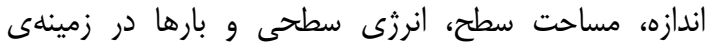

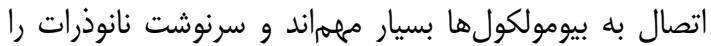

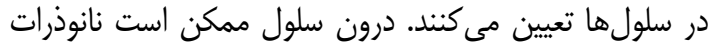

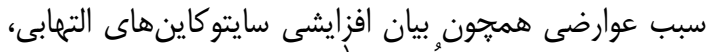

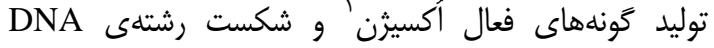
شوند. توليد بيش از حد

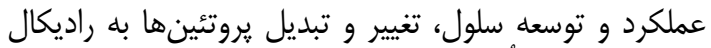

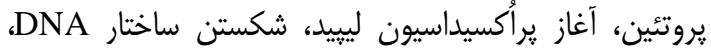

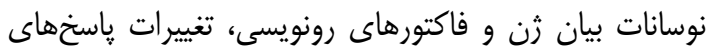

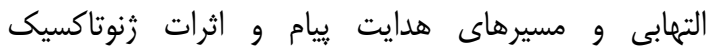

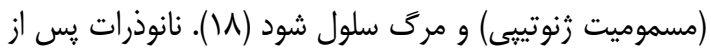

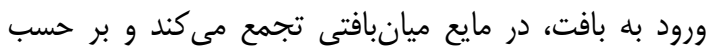

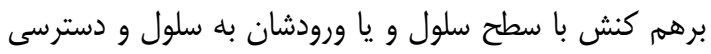

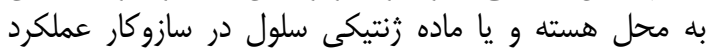

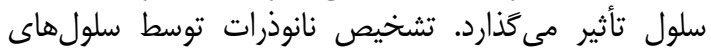

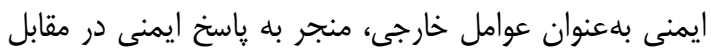

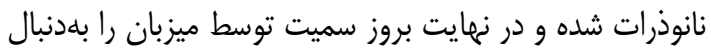

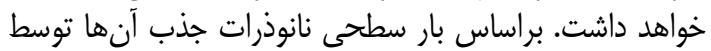

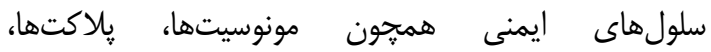

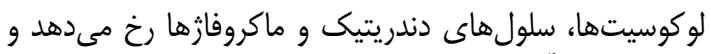

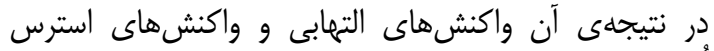

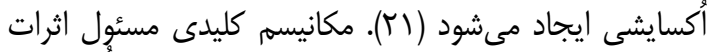

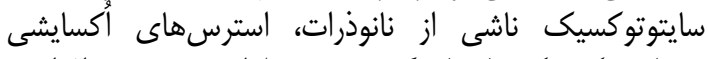

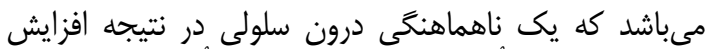

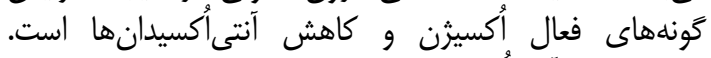

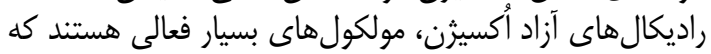

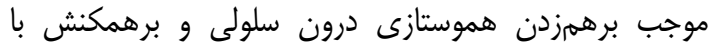

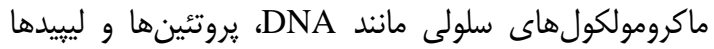

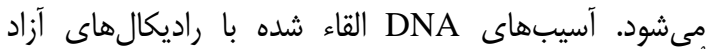

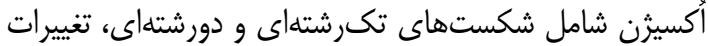

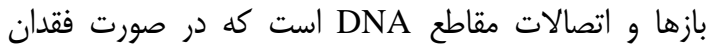

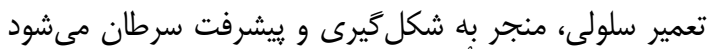

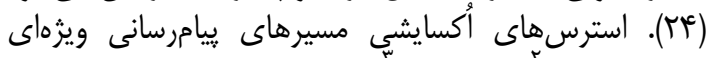

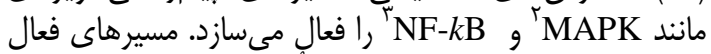

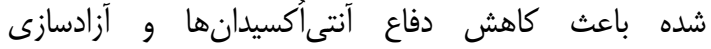

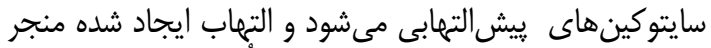

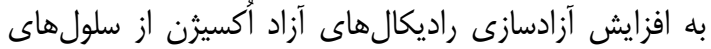

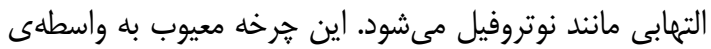

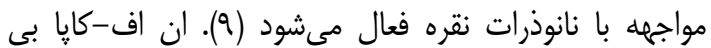
(NF-kB)

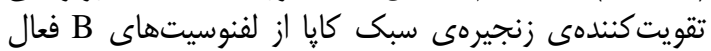

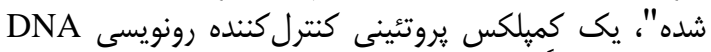

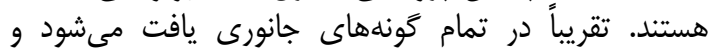

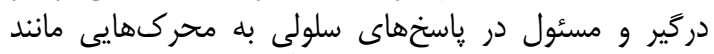

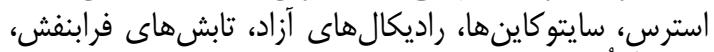

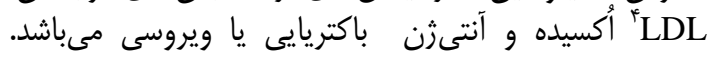

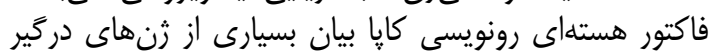


براى (Gallus gallus) طراحى شدند. ساخت آغازگر ها توسط

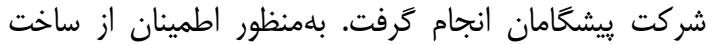
صحيح DNA مكمل، نمونههاى cDNA با استفاده از آغازگر

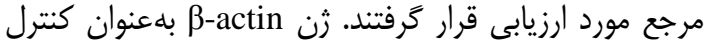

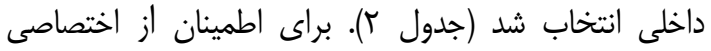

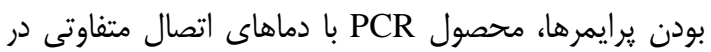

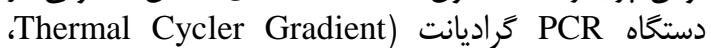

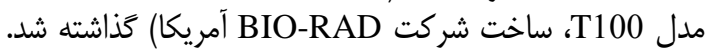

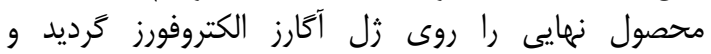

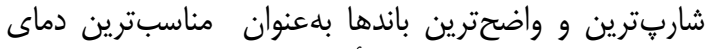

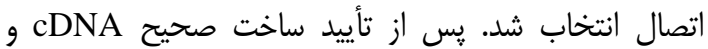

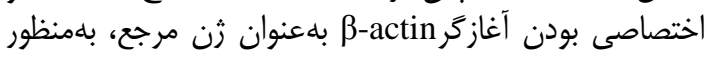

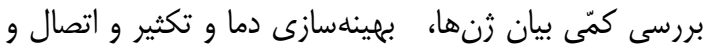

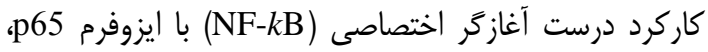

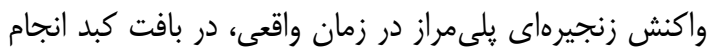

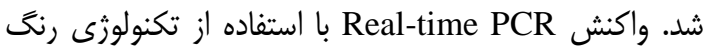

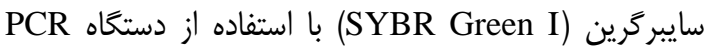
BIO- Thermal Cycler) آمريكا) و نرمافزار RAD إتيكال انجام شد. مخلوط واكنش شامل Primer Reverse Primer Forward SYBR Green ،DMSO DEPC- treated water

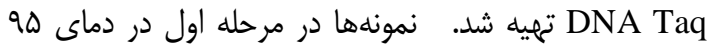

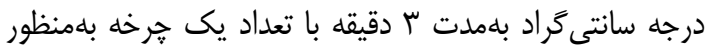

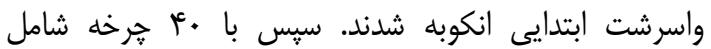

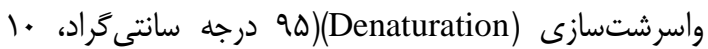

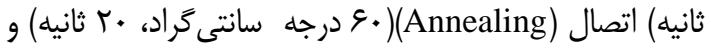

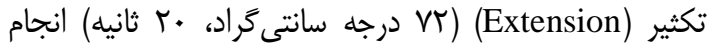

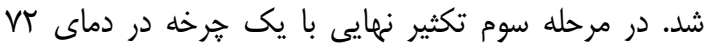
درجه سانتى گراد بهمدت ه د دقيقه انجام شد و د در انتها، مرحله

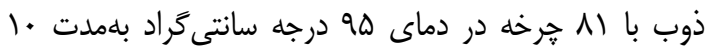

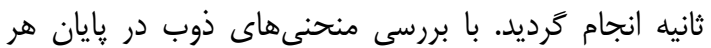

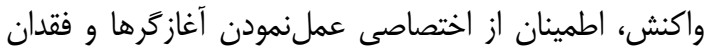

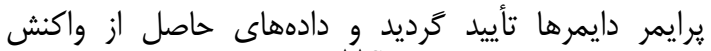

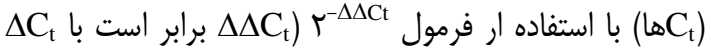

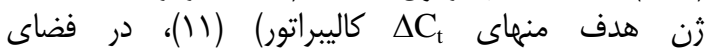

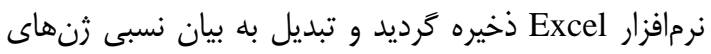

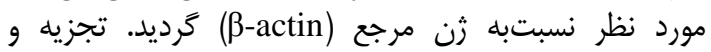

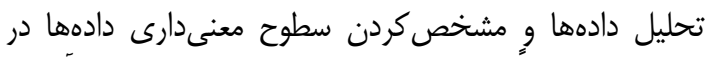

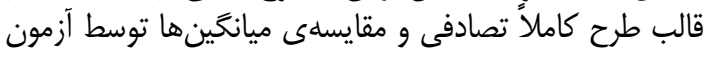

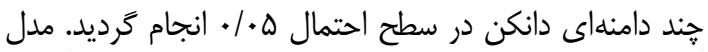

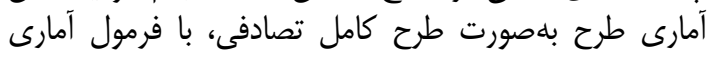
زير محاسبه كرديد.

$\mathrm{y}_{\mathrm{ij}}=\mu_{+} \alpha_{\mathrm{i}+} \varepsilon_{\mathrm{ij}}$

مقدار هر فراسنجه

ميانگين كل

$\alpha_{i=}$

خطاى آزمايش = ت

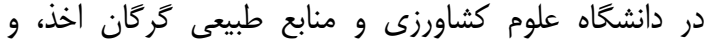

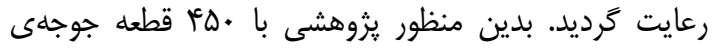

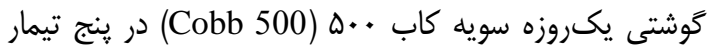

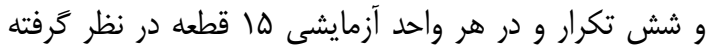

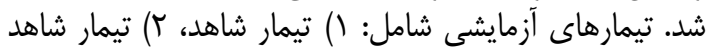

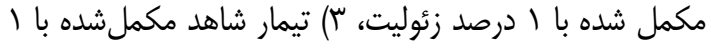

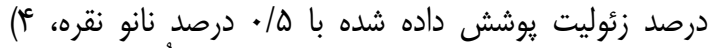

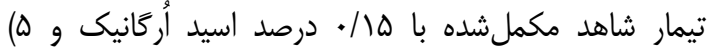

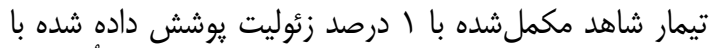

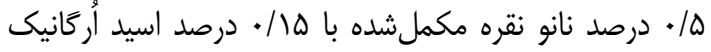

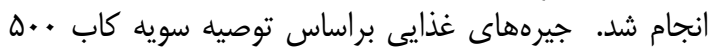

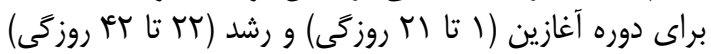

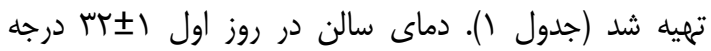

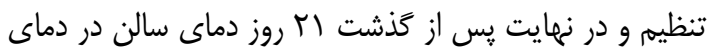

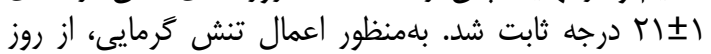

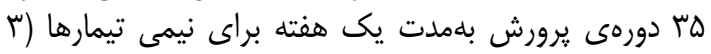

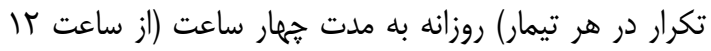

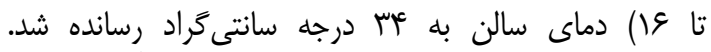

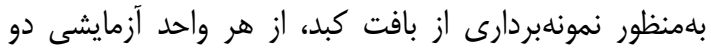

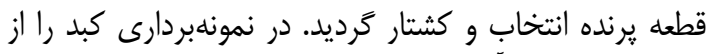

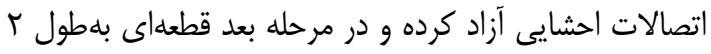

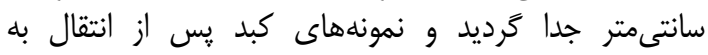

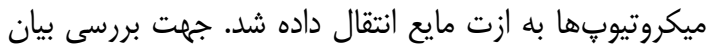

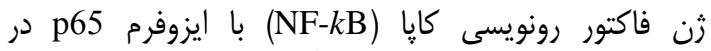

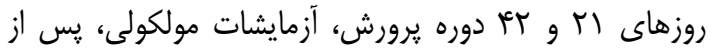

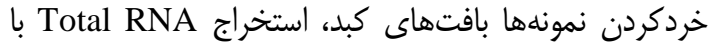

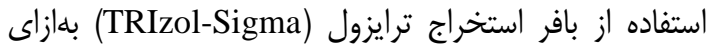

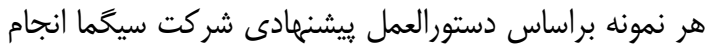

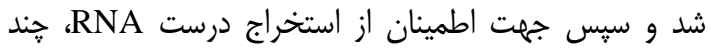

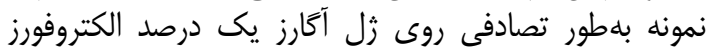

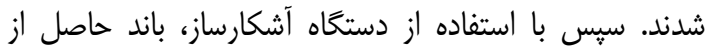

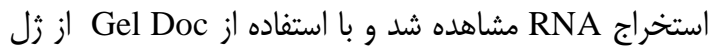

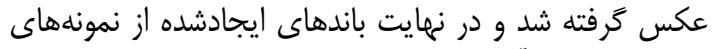

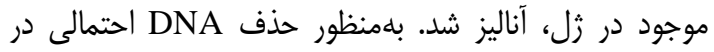

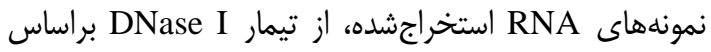

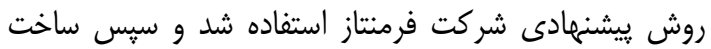

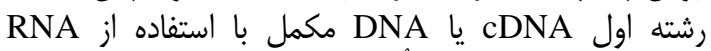

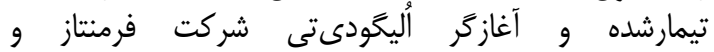
DEPC-treated water

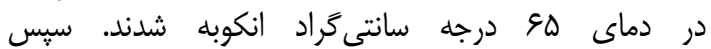
و Ribolock RNase Inhibitor،dNTP ،Buffer RT(5X)

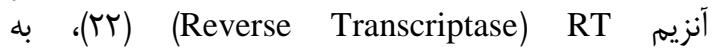

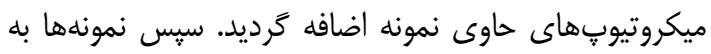

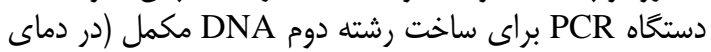

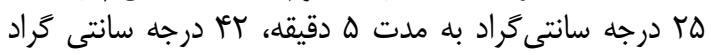

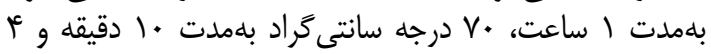

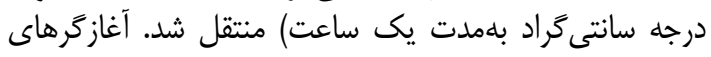

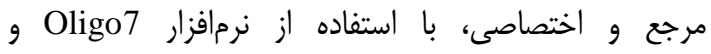

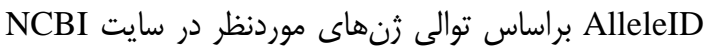


Table 1. Composition of experimental diets (DM basis) ${ }^{1}$

جدول (- تركيب جيرههاى غذايى (برحسب درصد ماده خشك')

\begin{tabular}{|c|c|c|}
\hline جيره رشد (Fr-FY) & جيره آغازين (الr- () & اجزاى جيره \\
\hline$\Delta Q / Q 9$ & $\Delta \Psi / V$ & ذرت \\
\hline Tr/Ta & $r q / \Delta r$ & كنجاله سويا \\
\hline$r / 4)$ & r & روغن سويا \\
\hline $1 / .9$ & $1 / 4 V$ & دى كلسيم فسفات \\
\hline $1 / \pi q$ & $1 / 19$ & سنگ آهى \\
\hline 的 & سז/א. & 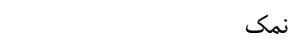 \\
\hline.$/ T \Delta$ & $\cdot / T \Delta$ & مكمل ويتامينى \\
\hline$\cdot / r \Delta$ & $\cdot / r \Delta$ & مكمل معدنى \\
\hline $.1 \cdot 0$ & 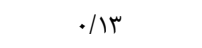 & DL متيونين DL \\
\hline.$/ 1 \%$ & .1 .9 & ليزين L \\
\hline & & آناليز مواد مغذى \\
\hline$r \cdot \omega \cdot$ & r9o. & انرزى قابل متابوليسم (Kcal/Kg) \\
\hline $19 / .9$ & $r / r$ & يروتئين خام (\%) \\
\hline$\cdot / N G$ &.$/ 94$ & كلسيم (\%) \\
\hline 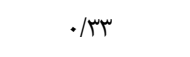 & $\cdot / 41$ & فسفر (\%) \\
\hline.$/ 14$ &.$/ 1 \Lambda$ & سديم (\%) \\
\hline.$/ 9 \Delta$ & $1 / \cdot 1$ & ليزين (\%) \\
\hline 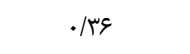 &.$/ 4 \mathrm{~V}$ & متيونين (٪) \\
\hline$\cdot / r v$ & 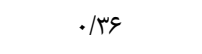 & سيستئين (\%) \\
\hline $1 / T V$ & $1 / E_{0}$ & آررثنين (\%) \\
\hline$\cdot / V^{4}$ & $\cdot / \Lambda F$ & ترئونين (\%) \\
\hline
\end{tabular}

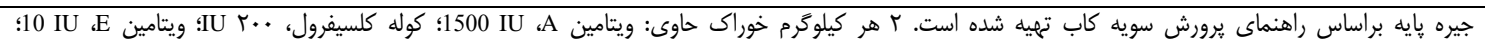

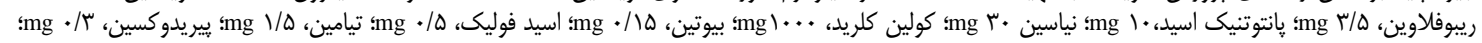

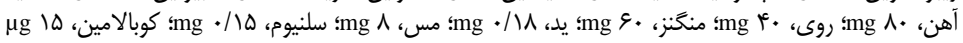

جدول r- اسامى ثنها و توالى يرايمرهاى مورد استفاده در واكنش qRT-PCR

Table 2. The primer sequences used in relative quantitative real-time PCR (q-PCR)

\begin{tabular}{|c|c|c|c|}
\hline 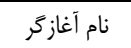 & توالى آغازگر & طول محصول (bp) & Access number \\
\hline NF- $k B(p 65)$ & $\begin{array}{l}\text { F: 5'- GTCCTCTCCCAGCCCATCTA-3' } \\
\text { R: 5'- TCAGCCCAGAAACGAACCTC-3' }\end{array}$ & 161 & XM-025145278 \\
\hline$\beta$-actin & $\begin{array}{l}\text { F: 5'- AAGTTACTCGCCTCTGTGAA- 3' } \\
\text { R: 5'- CACATCTATCACTGGGGAAC-3' }\end{array}$ & 198 & NM-205518 \\
\hline
\end{tabular}

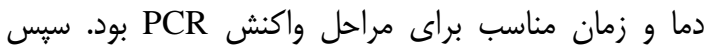

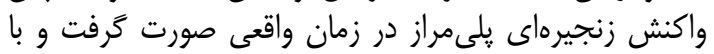

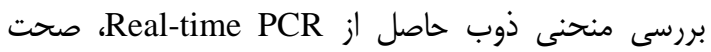

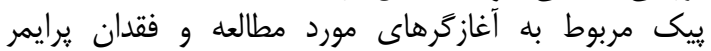

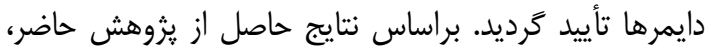

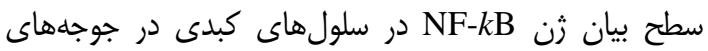

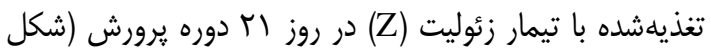

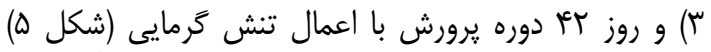

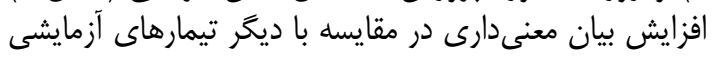

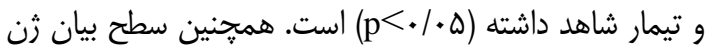
NF-kB

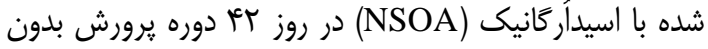

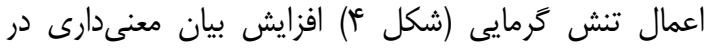

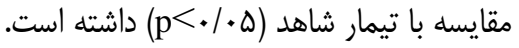

\section{نتايج و بحث}

كيفيت RNA، با قراردادن نمونههاى استخراجشده روى

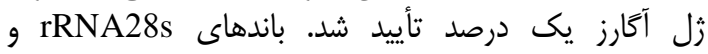

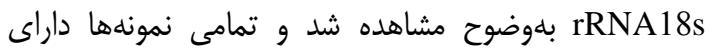

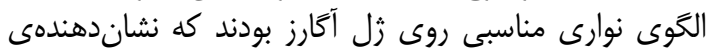

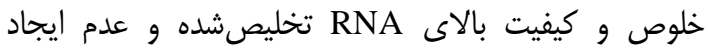

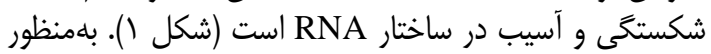

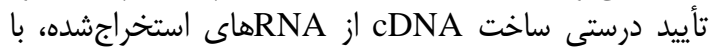

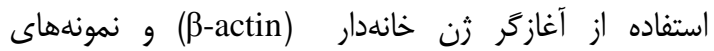
cDNA

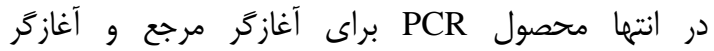

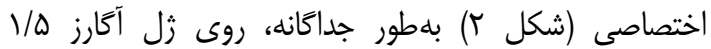

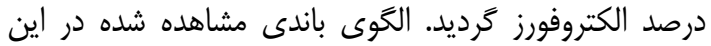

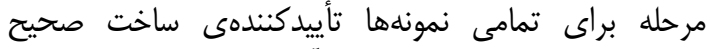

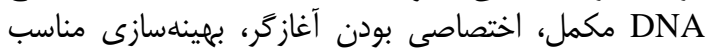




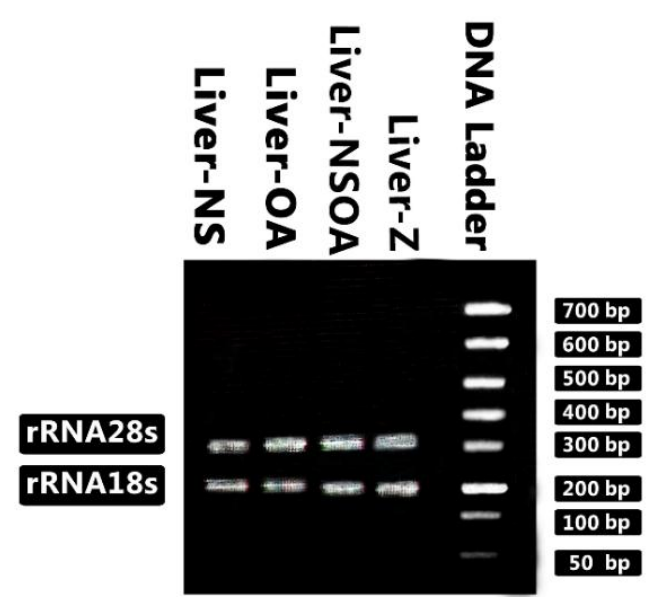

شكل RNA - استخراج شده از بافت كبد جوجههاى گوشتى

Figure 1. Extraction RNA from liver tissue of broiler chicken

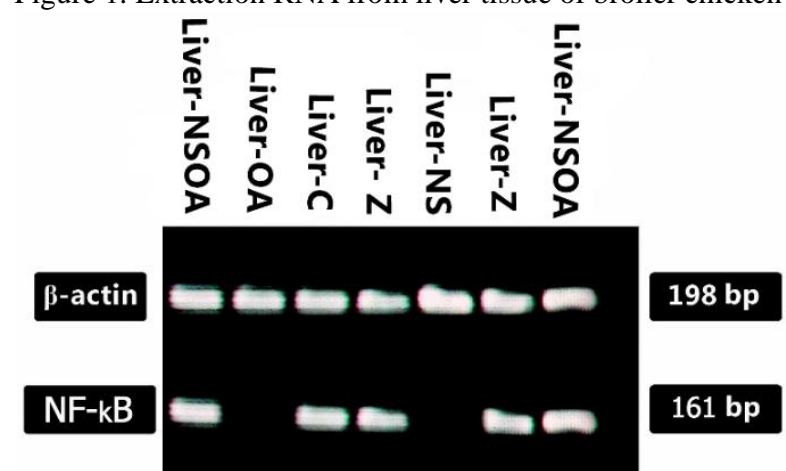

شكل r cDNA با استفاده از آغازگر ثن هاى مرجع و هدف در بافت كبد جوجههاى گوشتى Figure 2. Synthesized cDNA by specific primer for housekeeping and target gene in liver tissue of broiler chicken

\section{(NF-kB) Liver}

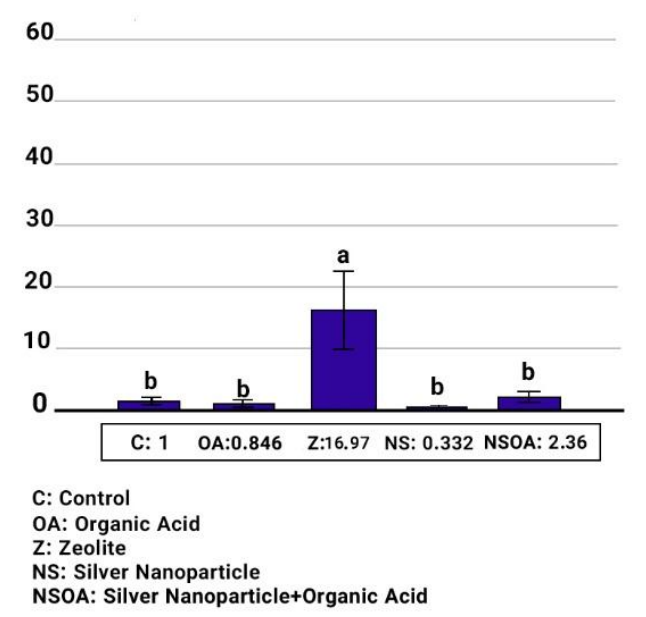

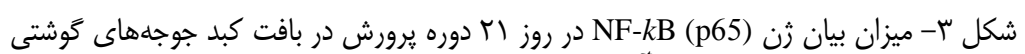

Figure 3. Expression of NF-kB (p65) gene at $21^{\text {st }}$ day of experiment in liver tissue of broiler chickens 


\section{(NF-kB) Liver}

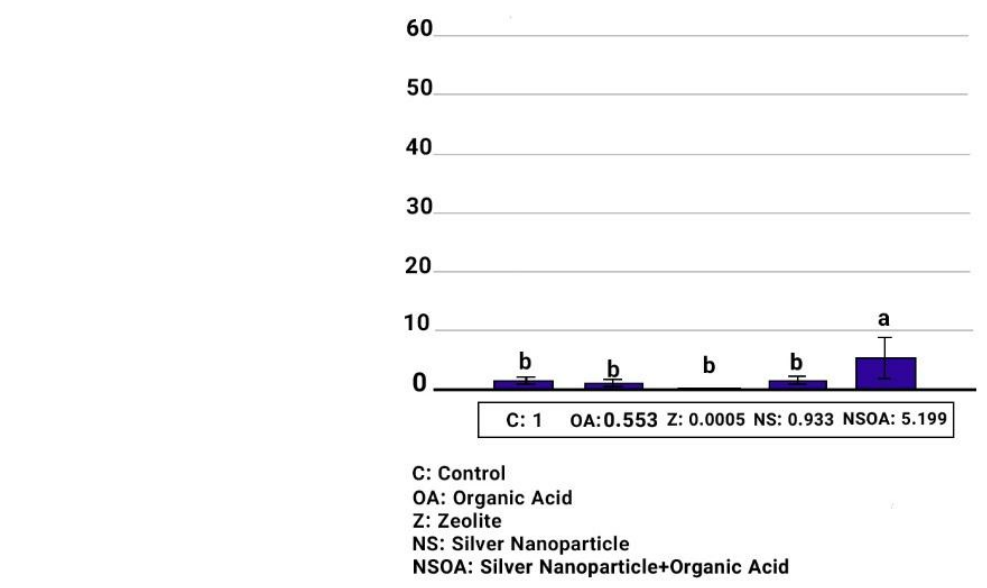

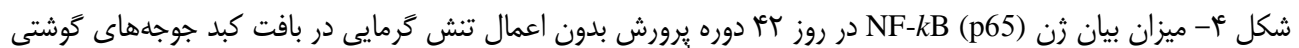

Figure 4. Expression of NF-kB (p65) gene at $42^{\text {nd }}$ day of experiment in liver tissue of broiler chickens without heat stress condition

\section{(NF-kB) Liver}

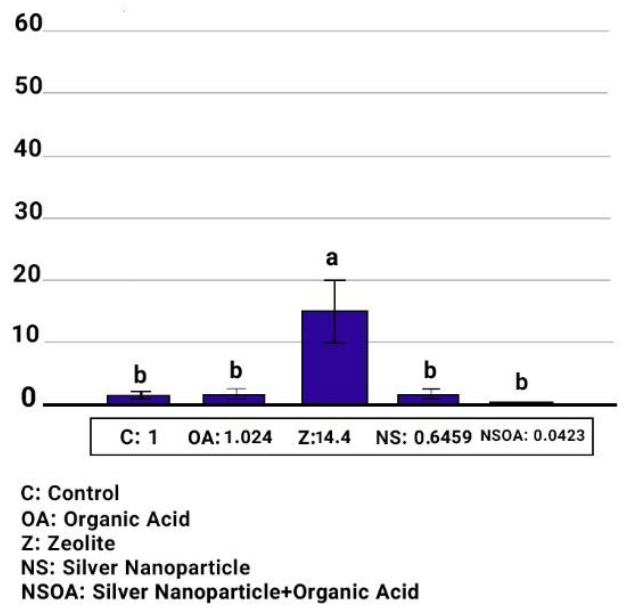

شكل ه- ميزان بيان ثن NF-kB (p65 در روز rأ دوره يرورش با اعمال تنش گرمايى در بافت كبد جوجههاى گوشتى

Figure 5. Expression of NF-kB (p65) gene at $42^{\text {nd }}$ day of experiment in liver tissue of broiler chickens with heat stress condition

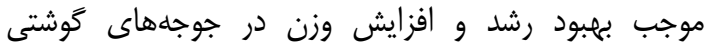

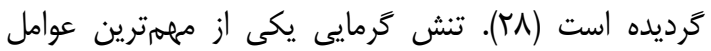

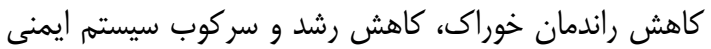

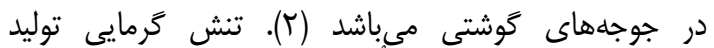

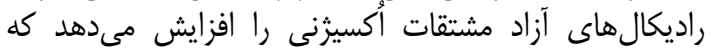

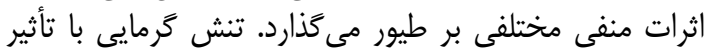

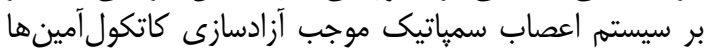

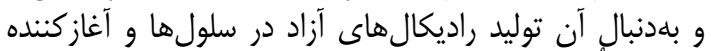

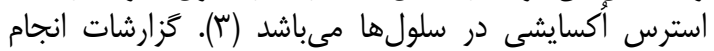

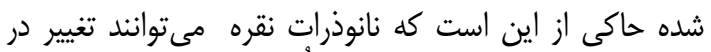

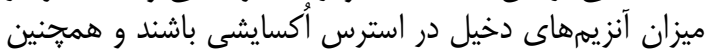

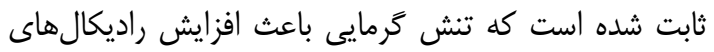

تغييرات مولكولى بهعنوان اولين تغييرات قابل اندازهگيرى

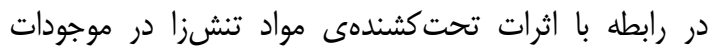

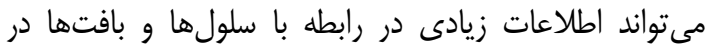

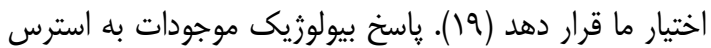

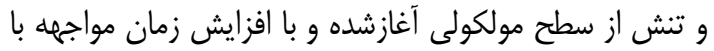
استرس، بلترتيب تغييرات در سطح بيوشيميايى، سلولى إنى، بافت،

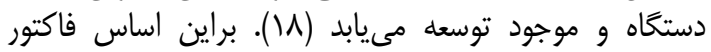

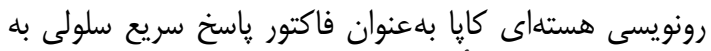

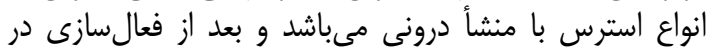

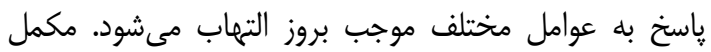
زئوليت بهعنوان حامل نانوذرات نقره در طيور استب استفاده مى إشود

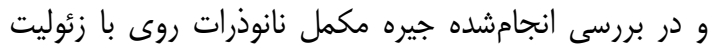




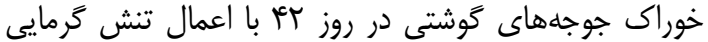

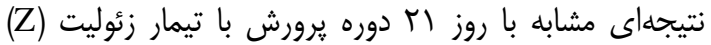

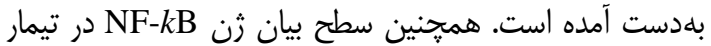

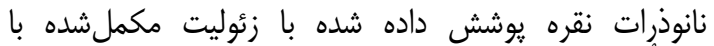

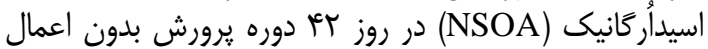

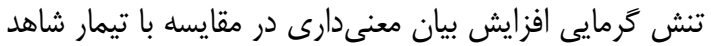

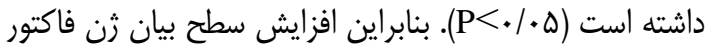

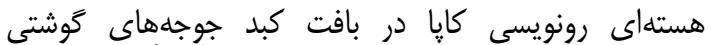

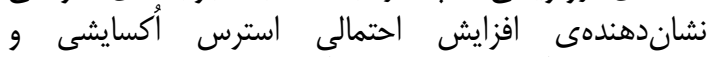
راديكالهاى آزاد و در نهايت آغاز و وراهاندازى مسيرهاى إنى

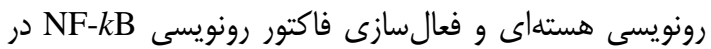

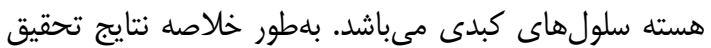
بيانَ آن است كه سلولهاى بافي مافت كبد تحت تأثير تيمارهاي

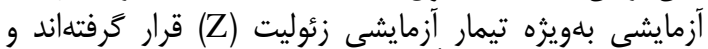

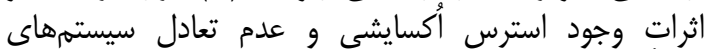

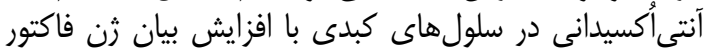

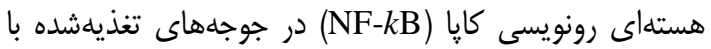

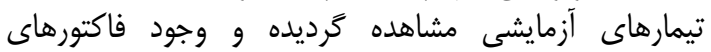

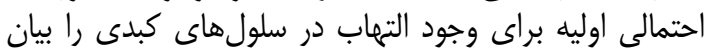

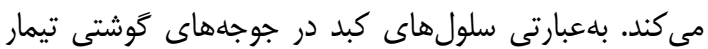

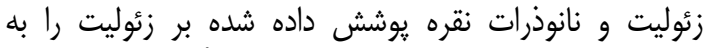

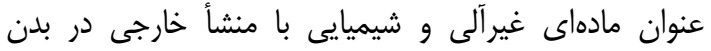

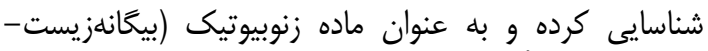

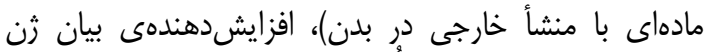

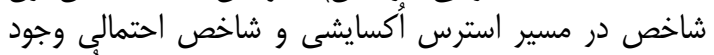

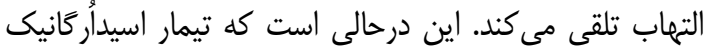

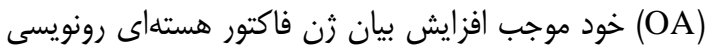

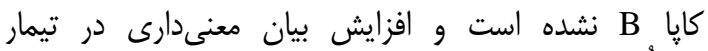

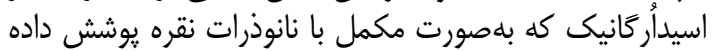
شده بر زئوليت (NSOA) مشاهده كرديده است.

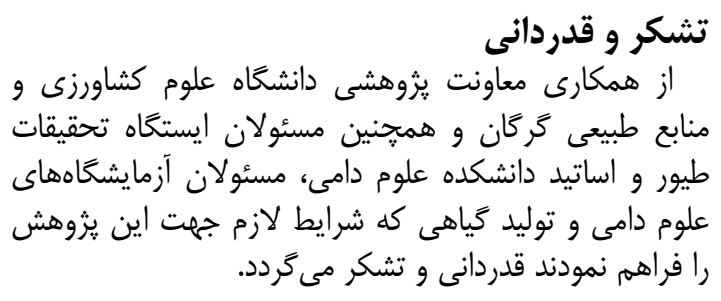

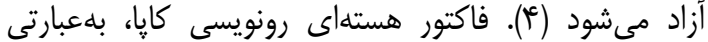

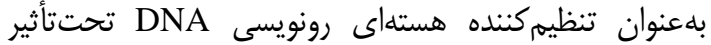
ROS

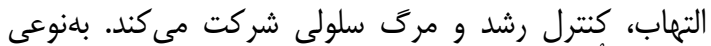

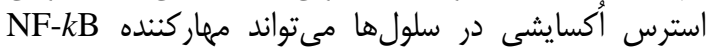
را تخريب كند و با تأثير (Inhibitory kappa B kinases)

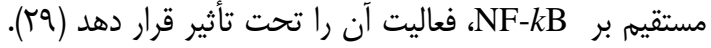

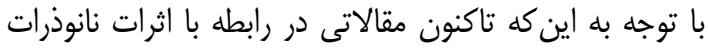

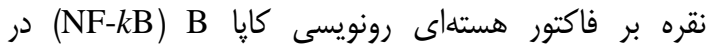

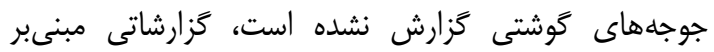

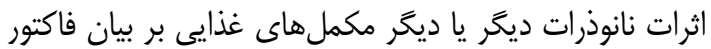

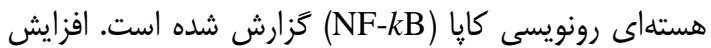

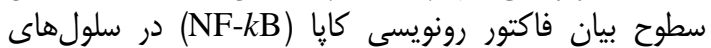
سرطانى كبدى انسان كه در معرض كابن نانيان

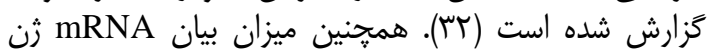
دF-kB

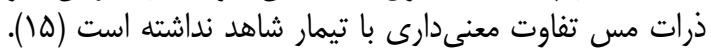

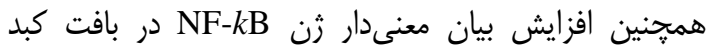

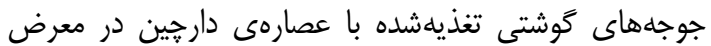
E. coli

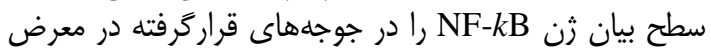

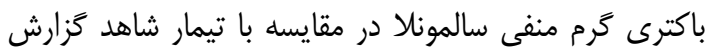

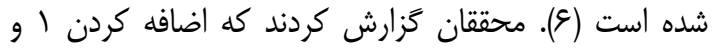

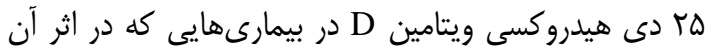

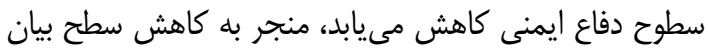

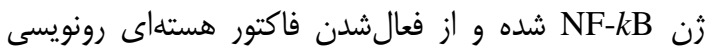

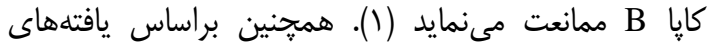

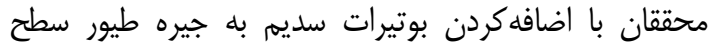

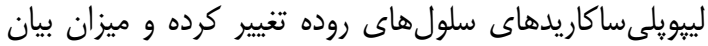
ر) تغن NF-kB

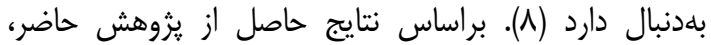

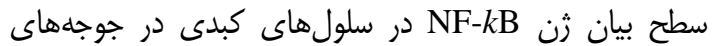

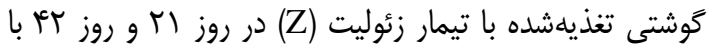

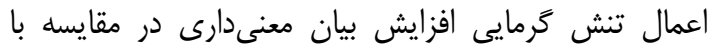

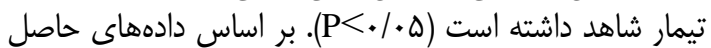

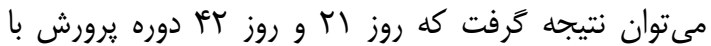

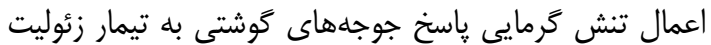

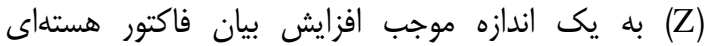
رونويسى كايا B كَرديده است و افزايش سن و افزايش مصرف

1. Chen, J. B. Bhandar and M. Kavdia. 2015. Interaction of ROS and RNS with GSH and GSH/GPX systems. The FASEB Journal, 29: 636-637.

2. Ciftci, M., U.G. Simsek, M.A. Azman, I.H. Cerci and F. Tonbak. 2013. The effects of dietary rosemary (Rosmarinus officinalis $l$ ) oil supplementation on performance, carcass traits and some blood parameters of Japanese quail under heat stressed condition. Ankara Universitesy Veteriner Fakültesi Dergisi, 19: 595-599.

3. Curi, R., P. Newsholme, M.M.R. Lima, T.C. Pithon-curi and J. Procopio. 2003. Glutamine and glutamate-their central role in cell metabolism and function. Cell Biochemistry and Function, 21: 1-9.

4. Esmaili, M., S.R. Hashemi, Y. Jafari Ahangari, S. Hassani and A. Shabani. 2017. Effect of different levels of silver nanoparticles coated with zeolite on performance, function of superoxide dismutase and glutathione peroxidase, carcass characteristics and internal organs weight of broiler chickens. Animal Production Research, 4: 1-11. 
5. Gilmore, T.D. 2006. Introduction to NF- $k$ B: Players, pathways, perspectives. Oncogene, 25: 66806684.

6. Hsin, Y.H., C.F. Chen, S. Huang and T.S. Shih. 2008. The apoptotic effect of nanosilver is mediated by a ROS-and JNK dependent mechanism involving the mitochondrial pathway in NIH3T3 cells. Toxicology Letters, 179:130-139.

7. Jiang, Z.Y., L.H. Sun, Y.C. Lin, X.Y. Ma, C.T. Zheng, G.L. Zhou, F. Chen and S.T. Zou. 2009. Effects of dietary glycyl-glutamine on growth performance, small intestinal integrity, and immune responses of weaning piglets challenged with lipopolysaccharide. Journal of Animal Science, 87: 4050-4056.

8. Jiang, Y., W. Zhang, F. Goa and G. Zhou. 2015. Effect of sodium butyrate on intestinal inflammatory response to lipopolysaccharide in broiler chickens. Canadian Journal of Animal Science, 95: 389-395.

9. Kreyling, W.G. 2010. A complementary definition of nanomaterial. Nano Today, 5: 165-168.

10. Li, Y., D.H. Chen, J. Yan, Y. Chen, R.A. Mittelstaedt, Y. Zhang, A.S. Biris, R.H. Heflich and T. Chen. 2012. Genotoxicity of silver nanoparticles evaluated using the Ames test and in vitro micronucleus assay. Mutation Research, 14: 4-10.

11. Livak, K.J. and T.D. Schmittgen. 2001. Analysis of relative gene expression data using real-time quantitative PCR and the $2^{-\Delta \Delta C r}$. Method, 25: 402-408.

12. Martirosyan, A., A. Bazes and Y.J. Schneider. 2014. In vitro toxicity assessment of silver nanoparticles in the presence of phenolic compounds-preventive agents against the harmful effect? Nanotoxicology, 8: 573-582.

13. Morones, J.R., J.L. Elechiguerra, A. Camacho and J.T. Ramirez. 2018. The bactericidal effect of silver nanoparticles. Nanotechnology, 16: 2346-2353.

14. Nagal, R. and R.K. Singla. 2013. Nanoparticles in different delivery systems: A brief review. Indo Global Journal of Pharmaceutical Sciences, 3: 96-106.

15. Oberdorster, G., V. Stone and K. Donaldson. 2007. Toxicology of nanoparticles: A historical perspective. Nanotoxicology, 1: 2-25.

16. Pinedaa, L., E. Sawoszb, K.P. Vadalasettya and A. Chwalibog. 2013. Effect of copper nanoparticles on metabolic rate and development of chicken embryos. Animal Feed Science and Technology, 186: 125-129.

17. Reiner, E., Z. Radic and V. Simeon-Rudolf. 2007. Mechanisms of organophosphate toxicity and detoxication with emphasis on studies in Croatia. Toxicity and Detoxification of Organophosphates, 58: 329-338.

18. Ribeiro, C.V., L. Belger, E. Pelletier and C. Rouleau. 2002. Histopathological evidence of inorganic mercury and methy lemercury toxicity in Arcitic charr (Salvelinus aipines). Environmental Research, 90: 217-225.

19. Rose, W.L., R.M. Nisbet, P.G. Green, S. Norris, T. Fan, E.H. Smith, G.N. Cherr and S.L. Anderson. 2006. Using an integrated approach to link biomarker responses and Physiological stress to growth impairment of cadmium-exposed larval topsmelt. Aquatic Toxicology, 80: 298-308.

20. Roy, R., R. Kumar and A. Tripathi. 2014. Intractive threats of nanoparticles to the biological system. Immunology Letters, 158: 79-87.

21. Salari-Joo, H.R., M.R. Kalbassi and S.A. Johari. 2012. Effect of water salinity on acute toxicity of colloidal silver nanoparticles in rainbow trout (Oncorhynchus mykiss) larvae. Iranian Journal of Health and Environment, 5: 121-132.

22. Sambrook, J. and D.W. Russel. 2001. Molecular cloning: a laboratory manual. New York, California, Academic Press, PP: 94-98.

23. Shoelson, S.E., J. Lee and M. Yuan. 2003. Inflammation and the IKK $\beta / \mathrm{I} \kappa \mathrm{B} / \mathrm{NF}-\kappa \mathrm{B}$ axis in obesityand diet-induced insulin resistance. International Journal of Obesity, 27: 49-52.

24. Singh, N., B. Manshian, G.J. Jenkins, S.M. Griffiths, P.M. Williams and T.G. Maffeis. 2009. Nano Genotoxicology: the DNA damaging potential of engineered nanomaterials. Biomaterials, 30: 38913914.

25. Smical, I. 2011. Properties of natural zeolites in benefit of nutrition and health. Human and Veterinary Medicine-Bioflux, 3: 51-57.

26. Susan, W.P.W., J.J.M. Willie, A.H. Carla, I.H. Werner, E.H.W. Heugens, B. Roszek, J. Bisschops and I. Gosens. 2009. Nano-silver-a review of available data and knowledge gaps in human and environmental risk assessment. Nanotoxicology, 3: 109-138.

27. Tabatabaei, S.M., R. Badalzade, GH.R. Mohammadnezhad and R. Balaei. 2015. Effects of Cinnamon extract on biochemical enzymes, TNF- $\alpha$ and NF- $\kappa \mathrm{B}$ gene expression levels in liver of broiler chickens inoculated with Escherichia coli. Pesquisa Veterinaria Brasileira, 35: 781-787.

28. Tang, Z.G., G.Y. Chen, L.F. Li, C. Wen, T. Wang and Y.M. Zhou. 2015. Effect of zinc-bearing zeolite clinoptilolite on growth performance, zinc accumulation, and gene expression of zinc transporters in broilers. American Society of Animal Science, 93:620-626.

29. Wijnhoven, S.W.P., W.J.G. Peijnenburg, C.A. Herberts, W.I. Hagens, A.G. Oomen and E.H.W. Heugens. 2009. Nanosilver: A review of available data and knowledge gaps in human and environmental risk assessment. Nanotoxicology, 3: 109-138.

30. Wu, Q.J., L.C. Wang, Y.M. Zhou, J.F. Zhang and T. Wang. 2013. Effects of clinoptilolite and modified clinoptilolite on the growth performance, intestinal microflora, and gut parameters of broilers. Poultry Science, 92: 684-692.

31. Zhang, K.Y., F. Yan, C.A. Keen and P.W. Waldroup. 2005. Evaluation of microencapsulated essential oils and organic acids in diets for broiler chickens. International Journal of Poultry Science, 4: 612619.

32.Zhang, X.F., W. Shen and S. Gurunathan. 2016. Silver nanoparticle-mediated cellular responses in various cell lines: An in vitro model. International Molecular Journal of Science, 17: 1-26. 


\title{
Evaluation of NF- $k$ B Gene Expression in liver tissue of Broiler Chickens fed with Silver Nanoparticles as an Indicator of Inflammation Induction in Heat Stress Conditions
}

\author{
EInaz Arabiyan ${ }^{1}$, Seyed Reza Hashemi ${ }^{2}$, Ahad Yamchi $^{3}$, Homa Davoodi $^{4}$ and \\ Sharif Rostami \\ 1- MSc. Student, Department of Animal Physiology, Faculty of Animal Science, Gorgan University of Agricultural \\ Sciences and Natural Resources, Gorgan, Iran \\ 2- Assistant Professor, Department of Animal Physiology, Faculty of Animal Science, Gorgan University of \\ Agricultural Sciences and Natural Resources, Gorgan, Iran \\ 3- Assistant Professor, Department of Plant Breeding and Biotechnology, Faculty of Plant Production, Gorgan \\ University of Agricultural Sciences and Natural Resources, Gorgan, Iran \\ 4- Assistant Professor, Department of Immunology, Faculty of Medical Science, Golestan University of Medical \\ Science, Gorgan, Iran \\ 5- PhD Student, Department of Animal Physiology, Faculty of Animal Science, Gorgan University of Agricultural \\ Sciences and Natural Resources, Gorgan, Iran \\ Received: January 15, $2019 \quad$ Accepted: June 2, 2019
}

\begin{abstract}
Heat stress is one of the most important immunosuppressive factors in poultry industry and heat stress has been associated with increases reactive oxygen species and oxidative stress in cells. Since the nuclear factor kappa-B is known as fast cellular response factor to endogenous stress; this study was conducted to evaluate relative gene expression in broiler chicken with 450 one-day-old Cobb 500 broiler chickens for 42 days. Treatments included: (1) basal diet (control), (2) basal diet containing 1\% zeolite, (3) basal diet containing 1\% of zeolite-coated with $0.5 \%$ silver nanoparticles, (4) Basal diet containing with $0.15 \%$ organic acids and (5) Basal diet containing $1 \%$ of zeolite-coated with $0.5 \%$ of silver nanoparticles and $0.15 \%$ organic acids in with or without heat stress condition. Liver biopsy was done in $21^{\text {st }}$ and $42^{\text {dd }}$ days of experiment. Results demonstrated the level of nuclear factor kappa-B relative gene expression in the $21^{\text {st }}$ and $42^{\text {nd }}$ days of experiment in heat stress condition in zeolite treatment was significant increase expression $(\mathrm{P}<0.05)$. Also in $42^{\text {nd }}$ days of experiment without heat stress condition in silver nanoparticles coated with zeolite in combination with organic acid treatment in broiler liver was significant increase expression comparison with control and all treatments $(\mathrm{P}<0.05)$. In conclusion results showed that zeolite and silver nanoparticles identified as an inorganic, chemical and exogenous substances in body tissues and liver identified this substances as a xenobiotics and up-regulated expression of index genes in oxidative stress pathways and inflammation. Whereas this effect is not identified in organic acid.
\end{abstract}

Keywords: Broiler, Immunity, Inflammation, NF- $k \mathrm{~B}$, Nano Silver 